\title{
On the $r$-dynamic coloring of some fan graph families
}

\author{
Raúl M. Falcón, M. Venkatachalam, S. Gowri and G. Nandini
}

\begin{abstract}
In this paper, we determine the $r$-dynamic chromatic number of the fan graph $F_{m, n}$ and determine sharp bounds of this graph invariant for four related families of graphs: The middle graph $M\left(F_{m, n}\right)$, the total graph $T\left(F_{m, n}\right)$, the central graph $C\left(F_{m, n}\right)$ and the line graph $L\left(F_{m, n}\right)$. In addition, we determine the $r$-dynamic chromatic number of each one of these four families of graphs in case of being $m=1$.
\end{abstract}

\section{Introduction}

Let $r$ and $\ell$ be two positive integers. In 2001, as a natural generalization of the classical problem of graph coloring, Montgomery [24] introduced the concept of $r$-dynamic proper $\ell$-coloring of a given graph $G=(V(G), E(G))$ as any proper $\ell$-coloring $c: V(G) \rightarrow\{0, \ldots, \ell-1\}$ such that every vertex $v \in V(G)$ satisfies that

$$
|c(N(v))| \geq \min \{r, d(v)\} .
$$

Here, $N(v)$ and $d(v)$ denote, respectively, the neighborhood and the degree of the vertex $v$. That is to say, in practice, the colors of the vertices within the neighborhood of each given vertex $v \in V(G)$ are pairwise distinct, whenever $r \geq d(v)$. Otherwise, there exist at least $r$ distinct colors in its neighborhood.

Key Words: $r$-dynamic coloring, fan graph, middle graph, total graph, central graph, line graph.

2010 Mathematics Subject Classification: Primary 05C15.

Received: 01.02.2021

Accepted: 15.05 .2021 
The minimum positive integer $\ell$ for which an $r$-dynamic proper $\ell$-coloring of $G$ exists constitutes the $r$-dynamic chromatic number $\chi_{r}(G)$ of the graph $G$. If $r=1$, then these two concepts coincide indeed with the classical ones of proper $\ell$-coloring and chromatic number of a graph. As a first stage in the study of this new graph coloring, the interest focused on the case $r=2$ [1-4]. It is in the recent literature where one may observe how the problem of computing $r$-chromatic numbers of distinct types and families of graphs, with $r>2$, has received particular interest from a wide amount of graph theorists and has become a prolific field of study $[6,8,9,11,15-17,20,26]$. Additionally, the study of upper bounds has also become a focus of study. Thus, for instance, Jahanbekam et al. [14] proved that $\chi_{r}(G) \leq r \cdot \Delta(G)+1$, for a general positive integer $r$. (Here, $\Delta(G)$ denotes the maximum degree of the vertices of $G$.) Previously, Lai et al. [19] had already proved that $\chi_{r}(G) \leq \Delta(G)+r^{2}-r+1$, whenever $\Delta(G) \leq r$.

Of particular relevance for the aim of this paper is the study of the $r$ dynamic coloring of fan graphs and related families [23,26], a type of graphs for which the study of different proper colorings awakes interest in the literature $[29,32,33]$. This paper delves into this topic by focusing on the study of the $r$-dynamic chromatic number of four families of graphs: middle graphs, total graphs, central graphs and line graphs, derived all of them from a given fan graph $F_{m, n}$.

In the recent literature, one can find studies concerning different proper colorings of middle, total, central and line graphs of different types of graphs $[5,27,28,30,31]$. More specifically, the $r$-dynamic proper coloring of these four families of graphs has already been studied for bistar graphs [25], gear graphs [7] and helm graphs [10]. Some partial results concerning fan graphs $F_{1, n}$ may be found in [23].

The $r$-dynamic chromatic number of the line graph of a given graph coincides with the $r$-dynamic edge chromatic number of the latter. Remind in this regard that Meganingtyas [21] introduced in 2015 the notion of $r$ dynamic proper k-edge-coloring of a graph $G=(V(G), E(G))$ as a proper $k$-edge-coloring $c$ such that

$$
|c(N(u v))| \geq \min \{r, d(u)+d(v)-2\},
$$

for every pair of adjacent vertices $u, v \in V(G)$, where $N(u v)$ denotes the set of edges that are incident to that one containing both vertices $u$ and $v$. The $r$-dynamic edge chromatic number $\lambda_{r}(G)$ is the minimum positive integer $k$ for which an $r$-dynamic proper $k$-edge-coloring of the graph $G$ exists. If $r=1$, then both concepts refer to the classical ones of proper edge-coloring and edge chromatic number. 
The paper is organized as follows. In Section 2, we describe some preliminary concepts and results on Graph Theory that are used throughout the manuscript. Then, Sections 3-6 deal, respectively, with the $r$-dynamic chromatic number of the middle, total, central and line graphs of a fan graph.

\section{Preliminaries}

This section deals with some preliminary concepts and results on Graph Theory that are used throughout the paper. For more details about this topic, we refer the reader to [13].

Any graph $G=(V(G), E(G))$ is formed by a set $V(G)$ of vertices and a set $E(G)$ of edges so that each edge contains two vertices, which are called adjacent. Two distinct edges sharing a common vertex are called incident. The cardinalities of $V(G)$ and $E(G)$ are, respectively, the order and size of $G$. If both values are finite, then $G$ is said to be finite. It is complete if any pair of vertices are adjacent. The complete graph of order $n$ is denoted $K_{n}$. The subset of vertices in $V(G)$ that are adjacent to a vertex $v \in V(G)$ is its neighborhood $N_{G}(v)$. The cardinality $d_{G}(v)$ of this set is its degree. Every vertex of degree zero is said to be isolated. From now on, we write $N(v)$ and $d(v)$ when there is no risk of confusion. In addition, $\delta(G)$ and $\Delta(G)$ denote, respectively, the minimum and maximum vertex degree of $G$. Further, a subgraph of $G$ is any graph $H$ such that $V(H) \subseteq V(G)$ and $E(H) \subseteq E(G)$. It is said to be induced if $E(H)$ is formed by all those edges in $E(G)$ connecting vertices of $V(H)$. It is an $n$-clique of $G$ if it coincides with $K_{n}$. Finally, a spanning subgraph of $G$ is any subgraph $H$ such that $V(H)=V(G)$.

From now on, let $v w \in E(G)$ denote the edge formed by two vertices $v, w \in V(G)$. It is a loop if $v=w$. Further, two distinct edges containing the same pair of vertices are said to be parallel. A graph is simple if it contains no loops and no parallel edges. From now on, all the graphs in this manuscript are simple and finite.

A path $P_{n}$ between two distinct vertices $v, w \in V(G)$ is any ordered sequence of $n$ adjacent and pairwise distinct vertices

$$
\left\langle v_{0}=v, v_{1}, \ldots, v_{n-2}, v_{n-1}=w\right\rangle
$$

in $V(G)$, with $n>2$. If $v=w$, then such a sequence is called a cycle, which is denoted $C_{n}$. The fan graph $F_{m, n}$ is the graph that results after joining each vertex of a set of $m$ isolated vertices with all the vertices of the path $P_{n}$.

The middle graph [12] of $G$ is the graph $M(G)$ having as vertices the set $V(M(G))=V(G) \cup E(G)$ so that two such vertices are adjacent if and only if they are either two incident edges in $G$ or a vertex and an edge in $G$ so that 
the latter contains the former. If besides an edge is added for each pair of adjacent vertices in $G$, then we get the total graph $T(G)$. Further, the central graph $C(G)$ derives from $G$ by replacing each edge of the latter with a path of size two and then joining each pair of non-adjacent vertices in $G$. Finally, the line graph $L(G)$ has the edges of $G$ as its set of vertices, so that two such vertices are adjacent if and only if the related two edges in $G$ are incident. Figure 1 illustrates these four graphs, all of them associated to $F_{2,3}$.

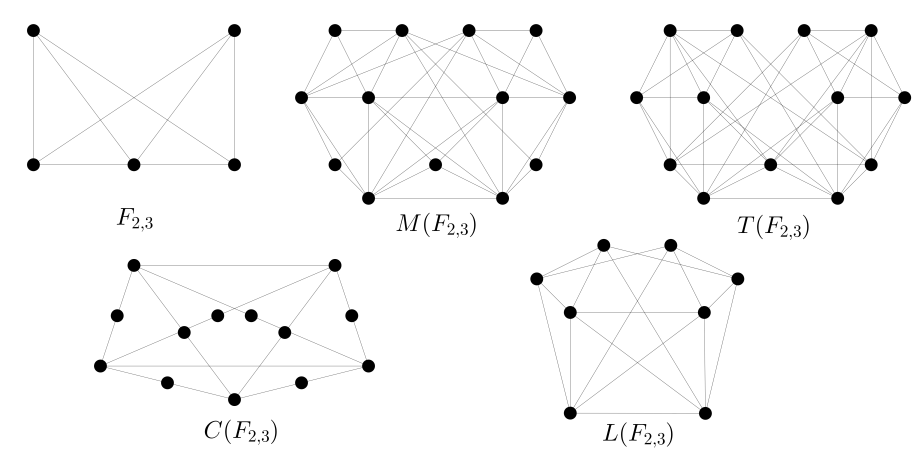

Figure 1: Fan graph, together with its middle, central, total and line graphs.

A proper $\ell$-coloring of the graph $G$ is any map $c: V(G) \rightarrow\{0, \ldots, \ell-1\}$ assigning a set of $\ell$ distinct colors to the set of vertices $V(G)$ in such a way that no two adjacent vertices share the same color. Analogously, a proper $\ell$-edge-coloring of the graph $G$ is any map $c: E(G) \rightarrow\{0, \ldots, \ell-1\}$ so that no two incident edges share the same color. The minimum positive integer $k$ for which a proper $k$-coloring (respectively, a proper $k$-edge-coloring) of the graph $G$ exists is termed the chromatic number $\chi(G)$ (respectively, the edge chromatic number $\lambda(G)$ ) of $G$. Particular examples are the $r$-dynamic proper $k$-(edge-)coloring and the $r$-dynamic (edge) chromatic number of a graph $G$, which have already been introduced in Section 1. Particularly, $\chi_{1}(G)=\chi(G)$, $\lambda_{1}(G)=\lambda(G)$ and $\lambda_{r}(G)=\chi_{r}(L(G))$, for every positive integer $r$.

The following results are also known.

Lemma 1. [18] Let $G$ be a graph and let $r$ be a positive integer. Then, $\min \{r, \Delta(G)\}+1 \leq \chi_{r}(G) \leq \chi_{r+1}(G)$. Moreover, $\chi_{r}(G) \leq \chi_{\Delta(G)}(G)$.

Lemma 2. [19] Let $n$ and $r$ be two positive integers such that $n>2$. Then,

a) $\chi_{r}\left(P_{n}\right)= \begin{cases}2, & \text { if } r=1, \\ 3, & \text { otherwise. }\end{cases}$ 


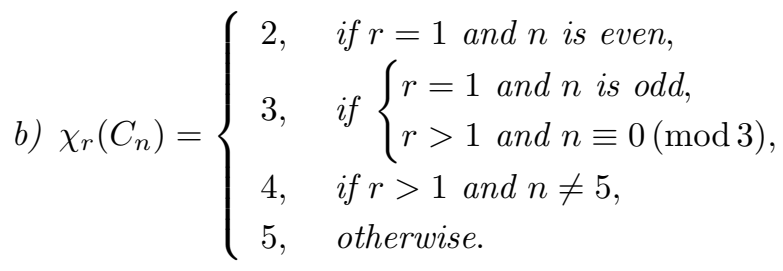

c) $\chi_{r}\left(K_{n}\right)=n$.

Lemma 3. [23] Let $n$ and $r$ be two positive integers such that $n>2$. Then,

a) $\chi_{2}\left(M\left(F_{1, n}\right)\right)=n+1$, if $n \geq 7$.

b) $\chi_{5}\left(T\left(F_{1, n}\right)\right)=n+1$, if $n \geq 9$.

c) $\chi_{2}\left(C\left(F_{1, n}\right)\right)=n+1$, if $n \geq 4$.

d) $\chi_{n}\left(L\left(F_{1, n}\right)\right)=n+1$, if $n \geq 6$.

Let $m$ and $n$ be two positive integers such that $n>2$. Throughout the whole paper, let $F_{m, n}$ denote the fan graph of set of vertices

$$
V\left(F_{m, n}\right)=\left\{u_{0}, \ldots, u_{m-1}, v_{0}, \ldots, v_{n-1}\right\},
$$

which is associated to the path $P_{n}=\left\langle v_{0}, \ldots, v_{n-1}\right\rangle$. That is, the set of edges of the fan graph $F_{m, n}$ is $E\left(F_{m, n}\right)=\left\{u_{0} v_{0}, \ldots, u_{0} v_{n-1}, \ldots, u_{m-1} v_{n-1}, v_{0} v_{1}, \ldots\right.$, $\left.v_{n-2} v_{n-1}\right\}$. In particular, $\delta\left(F_{m, n}\right)=\min \{m+1, n\}$ and $\Delta\left(F_{m, n}\right)=\max \{m+$ $2, n\}$. The following result establishes the $r$-dynamic coloring of the fan graph $F_{m, n}$.

Proposition 1. Let $m, n$ and $r$ be three positive integers such that $n>2$. Then,

$$
\chi_{r}\left(F_{m, n}\right)= \begin{cases}3, & \text { if } r \in\{1,2\}, \\ 2 r-1, & \text { if } 3 \leq r \leq \min \{m+1, n\}, \\ n+r-1, & \text { if } n<r \leq m+1, \\ m+r, & \text { if } \max \{3, m+1\} \leq r \leq n, \\ m+n, & \text { if } r \geq \max \{m+1, n\} .\end{cases}
$$

Proof. Since the vertices $u_{0}, v_{0}, v_{1} \in V\left(F_{m, n}\right)$ describe a 3-clique of $F_{m, n}$, Lemma 2 implies that every proper coloring of $F_{m, n}$ requires at least three distinct colors. In addition, since $N\left(u_{i}\right)=\left\{v_{j}: 0 \leq j<n\right\}$, for all nonnegative integer $i<m$, and $N\left(v_{0}\right)=\left\{u_{i}: 0 \leq i<m\right\} \cup\left\{v_{1}\right\}$, the remaining values constitute lower bounds of $\chi_{r}\left(F_{m, n}\right)$ under the corresponding assumptions because of Condition (1) and the fact that the map $c$ is a proper coloring. 
In order to prove that each one of these lower bounds is reached, it is enough to describe for each case an appropriate $r$-dynamic proper coloring $c$ of the fan graph $F_{m, n}$ satisfying Condition (1). Thus, for instance, if $r \in\{1,2\}$, then we define the map $c$ so that $c\left(u_{i}\right)=2$ and $c\left(v_{j}\right)=j \bmod 2$, for all non-negative integers $i<m$ and $j<n$. Furthermore, if $r \geq 3$, the map $c$ is defined so that, for each pair of non-negative integers $i<m$ and $j<n$, we have that

$$
c\left(u_{i}\right)= \begin{cases}\min \{r, n\}+i, & \text { if } i<\min \{r-1, m\} \\ \min \{r, n\}+\min \{r-1, m\}-1, & \text { otherwise. }\end{cases}
$$

and

$$
c\left(v_{j}\right)= \begin{cases}j, & \text { if } j<\min \{r, n\} \\ j \bmod r, & \text { otherwise }\end{cases}
$$

Figures 2 and 3 illustrate Proposition 1 for the fan graphs $F_{2,3}$ and $F_{6,4}$.
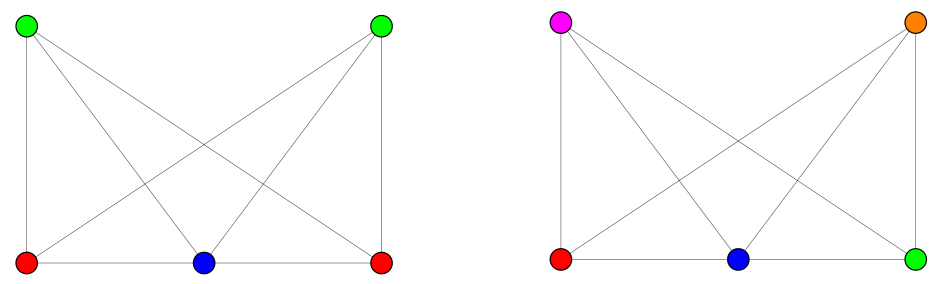

Figure 2: $r$-dynamic proper $\ell$ colorings of the fan graph $F_{2,3}$, for $(r, \ell) \in$ $\{1,2\} \times\{3\}$ (left) and $r \geq 3$ and $\ell=5$ (right).
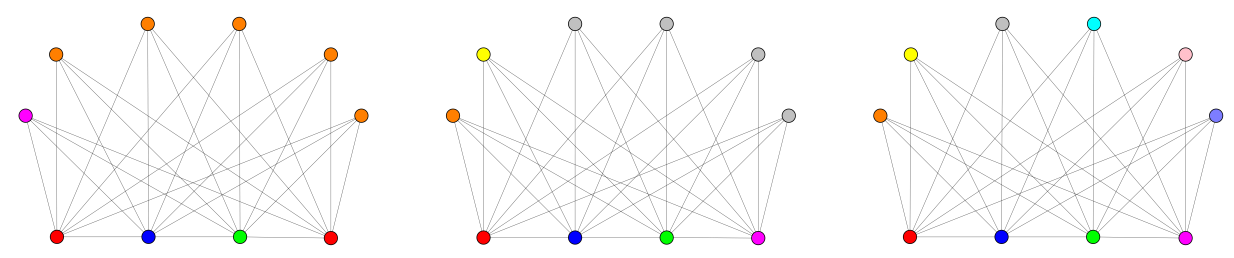

Figure 3: $r$-dynamic proper $\ell$-coloring of the fan graph $F_{6,4}$, for $(r, \ell)=(3,5)$ (left), $(r, \ell)=(4,7)$ (center) and $r \geq 7$ and $\ell=10$ (right). 


\section{Dynamic coloring of the middle graph of a fan graph}

This section deals with the $r$-dynamic chromatic number of the middle graph $M\left(F_{m, n}\right)$. Its set of vertices is

$$
\begin{aligned}
V\left(M\left(F_{m, n}\right)\right)=\{ & u_{0}, \ldots, u_{m-1}, v_{0}, \ldots, v_{n-1}, u_{0} v_{0}, \ldots, u_{0} v_{n-1}, \ldots, \\
& \left.u_{m-1} v_{0}, \ldots, u_{m-1} v_{n-1}, v_{0} v_{1}, \ldots, v_{n-2} v_{n-1}\right\}
\end{aligned}
$$

In this middle graph, for each pair of non-negative integers $i<m$ and $j<n$, we have that

$$
d\left(u_{i}\right)=n, \quad d\left(v_{j}\right)= \begin{cases}m+1, & \text { if } j \in\{0, n-1\}, \\ m+2, & \text { otherwise }\end{cases}
$$

and

$$
d\left(u_{i} v_{j}\right)= \begin{cases}m+n+1, & \text { if } j \in\{0, n-1\} \\ m+n+2, & \text { otherwise. }\end{cases}
$$

and, for each non-negative integer $k<n-1$, we have that

$$
d\left(v_{k} v_{k+1}\right)= \begin{cases}2 m+3, & \text { if } k \in\{0, n-2\}, \\ 2 m+4, & \text { otherwise. }\end{cases}
$$

Hence, $\delta\left(M\left(F_{m, n}\right)\right)=\min \{m+1, n\}$ and $\Delta\left(M\left(F_{m, n}\right)\right)=\max \{m+n+$ $2,2 m+4\}$. Further, for each pair of non-negative integers $i<m$ and $j<n$, let $K_{M\left(F_{m, n}\right)}(i)$ denote the $(n+1)$-clique of the middle graph $M\left(F_{m, n}\right)$ that is defined by the set of vertices

$$
V\left(K_{M\left(F_{m, n}\right)}(i)\right)=\left\{u_{i}, u_{i} v_{0}, \ldots, u_{i} v_{n-1}\right\}
$$

and let $K_{M\left(F_{m, n}\right)}^{\prime}(j)$ denote the induced subgraph of $M\left(F_{m, n}\right)$ that is defined by the set of vertices

$$
\begin{gathered}
\qquad\left\{\left(K_{M\left(F_{m, n}\right)}^{\prime}(j)\right)=\right. \\
= \begin{cases}\left\{v_{0}, u_{0} v_{0}, \ldots, u_{m-1} v_{0}, v_{0} v_{1}\right\}, & \text { if } j=0, \\
\left\{v_{n-1}, u_{0} v_{n-1}, \ldots, u_{m-1} v_{n-1}, v_{n-2} v_{n-1}\right\}, & \text { if } j=n-1, \\
\left\{v_{j}, u_{0} v_{j}, \ldots, u_{m-1} v_{j}, v_{j-1} v_{j}, v_{j} v_{j+1}\right\}, & \text { otherwise. }\end{cases}
\end{gathered}
$$

Notice that $K_{M\left(F_{m, n}\right)}^{\prime}(j)$ constitutes an $(m+2)$-clique within $M\left(F_{m, n}\right)$, if $j \in$ $\{0, n-1\}$, and an $(m+3)$-clique, otherwise. These cliques enable us to establish a pair of sharp bounds for the $r$-dynamic chromatic number $\chi_{r}\left(M\left(F_{m, n}\right)\right)$. 
Proposition 2. Let $m, n$ and $r$ be three positive integers such that $n>2$. Then,

$$
\max \{m+3, n+1\} \leq \chi_{r}\left(M\left(F_{m, n}\right)\right) \leq \begin{cases}3 m+5, & \text { if } n=3, \\ m \cdot n+6, & \text { otherwise. }\end{cases}
$$

The lower bound is reached for every positive integer $r<\max \{m+3, n+1\}$, whereas the upper bound is reached for every positive integer $r \geq \Delta\left(M\left(F_{m, n}\right)\right)$.

Proof. The lower bound holds simply from Lemma 2, once it is noticed that both the $(n+1)$-clique $K_{M\left(F_{m, n}\right)}(0)$ and the $(m+3)$-clique $K_{M\left(F_{m, n}\right)}^{\prime}(1)$ are subgraphs of the middle graph $M\left(F_{m, n}\right)$. In order to prove that this lower bound is sharp, let us describe an appropriate proper $\chi_{r}\left(M\left(F_{m, n}\right)\right)$-coloring $c$ of the middle graph $M\left(F_{m, n}\right)$ satisfying Condition (1). In order to make clearer the proof, we illustrate this map $c$ with a specific dynamic coloring of a middle graph $M\left(F_{m, n}\right)$, where the edges of the $(n+1)$-clique $K_{M\left(F_{m, n}\right)}(0)$ and the $(m+3)$-clique $K_{M\left(F_{m, n}\right)}^{\prime}(1)$ are respectively highlighted with bold and dashed lines. In addition, the vertices of the sets $\left\{u_{0}, \ldots, u_{m-1}\right\},\left\{v_{0}, \ldots, v_{n-1}\right\}$, $\left\{u_{0} v_{0}, \ldots, u_{m-1} v_{n-1}\right\}$ and $\left\{v_{0} v_{1}, \ldots, v_{n-2} v_{n-1}\right\}$ are respectively distinguished by means of the symbols $\boldsymbol{\Lambda}, \mathbf{\nabla}, \bullet$ and $\downarrow$. The following study of cases arises.

- Case $m \leq n-2$ and $r \leq n$.

Let the map $c$ be defined so that, for each pair of non-negative integers $i<m$ and $j<n$, we have that

$$
\begin{gathered}
c\left(u_{i}\right)=(n+i) \bmod (n+1), \quad c\left(v_{j}\right)=(2+j) \bmod (n+1), \\
c\left(u_{i} v_{j}\right)=(i+j) \bmod (n+1)
\end{gathered}
$$

and, for each non-negative integer $k<n-1$, we have that

$$
c\left(v_{k} v_{k+1}\right)=(n+k) \bmod (n+1) .
$$

Condition (1) holds and hence, $\chi_{r}\left(M\left(F_{m, n}\right)\right)=n+1$. Figure 4 illustrates the middle graph $M\left(F_{1,4}\right)$.

- Case $m>n-2$ and $r \leq m+2$.

Let the map $c$ be defined so that, for each pair of non-negative integers $i<m$ and $j<n$, we have that

$$
c\left(u_{i}\right)=m+1, \quad c\left(v_{j}\right)=(n+j-1) \bmod (m+1)
$$

and

$$
c\left(u_{i} v_{j}\right)=(i+j) \bmod (m+1),
$$




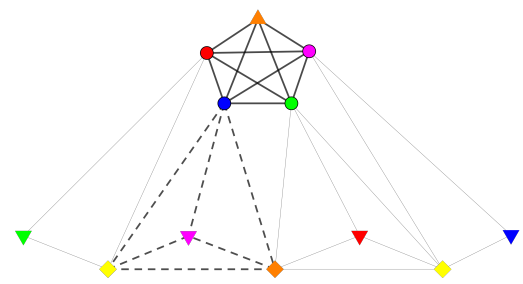

Figure 4: $r$-dynamic proper 5-coloring of $M\left(F_{1,4}\right)$, for all $r \leq 4$.

and, for each non-negative integer $k<n-1$, we have that

$$
c\left(v_{k} v_{k+1}\right)= \begin{cases}m+1, & \text { if } k \text { is even, } \\ m+2, & \text { otherwise. }\end{cases}
$$

Condition (1) holds and hence, $\chi_{r}\left(M\left(F_{m, n}\right)\right)=m+3$. Figure 5 illustrates the middle graph $M\left(F_{3,4}\right)$.

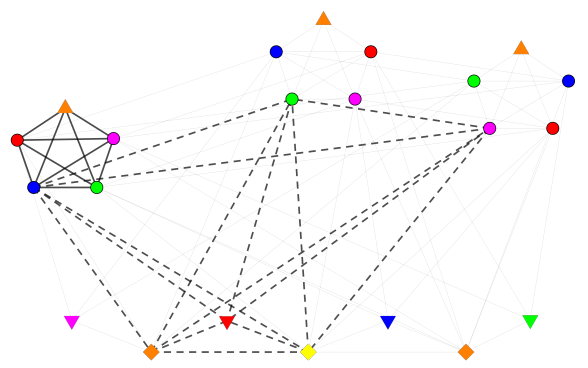

Figure 5: $r$-dynamic proper 6-coloring of $M\left(F_{3,4}\right)$, for all $r \leq 5$.

Now, in order to study the upper bound, Lemma 1 enables us to focus on the case $r=\Delta\left(M\left(F_{m, n}\right)\right)$. Let $c$ be a $\Delta\left(M\left(F_{m, n}\right)\right)$-dynamic proper $\chi_{\Delta\left(M\left(F_{m, n}\right)\right)}\left(M\left(F_{m, n}\right)\right)$-coloring of the middle graph $M\left(F_{m, n}\right)$. Then, Condition (1) implies that $|c(N(v))|=d(v)$, for all $v \in V\left(M\left(F_{m, n}\right)\right)$. In particular, the set $\left\{c\left(u_{0} v_{0}\right), \ldots, c\left(u_{m-1} v_{n-1}\right)\right\}$ is formed by $m \cdot n$ distinct colors, because $\left\{u_{i} v_{0}, \ldots, u_{i} v_{n-1}\right\} \subset V\left(K_{M\left(F_{m, n}\right)}(i)\right)$, for every non-negative integer $i<m$, and, if $c\left(u_{i} v_{j}\right)=c\left(u_{i^{\prime}} v_{j^{\prime}}\right)$ for some non-negative integers $i, i^{\prime}<m$ and $j, j^{\prime}<n$ such that $i \neq i^{\prime}$ and $j \neq j^{\prime}$, then we would have, for instance, that $\left|c\left(N\left(u_{i} v_{j^{\prime}}\right)\right)\right|<d\left(u_{i} v_{j^{\prime}}\right)$. In a similar way, each one of the three vertices $v_{1}$, $v_{0} v_{1}, v_{1} v_{2}$ of the $(m+3)$-clique $K_{M\left(F_{m, n}\right)}^{\prime}(1)$ requires an extra new color. 
Then, a simple study of the neighborhood $N\left(v_{0} v_{1}\right)$ implies that the vertex $v_{0}$ requires an extra $(m \cdot n+4)^{\text {th }}$ color. Similarly, if $n>3$, then a simple study of the neighborhood $N\left(v_{1} v_{2}\right)$ implies that either the vertex $v_{2}$ or the vertex $v_{2} v_{3}$ requires an extra $(m \cdot n+5)^{\text {th }}$ color. With only this palette of colors, any coloring of the vertex $u_{0}$ would contradict either Condition (1) or the fact that the map $c$ is a proper coloring. Thus, a new extra color is required for that vertex. In short, $3 m+5 \leq \chi_{\Delta\left(F_{m, 3}\right)}\left(F_{m, 3}\right)$ and, if $n>3$, then $m \cdot n+6 \leq \chi_{\Delta\left(M\left(F_{m, n}\right)\right)}\left(M\left(F_{m, n}\right)\right)$.

In order to prove that these lower bounds are indeed reached, it is enough to consider the following proper coloring $c$ of the middle graph $M\left(F_{m, n}\right)$ satisfying Condition (1). Particularly, for each pair of non-negative integers $i<m$ and $j<n$, we consider that

$c\left(u_{i}\right)=m \cdot n, \quad c\left(v_{j}\right)=m \cdot n+1+(j \bmod 2) \quad$ and $\quad c\left(u_{i} v_{j}\right)=i \cdot n+j$, and, for each non-negative integer $k<n-1$, we have that

$$
c\left(v_{k} v_{k+1}\right)=m \cdot n+3+(k \bmod 3) .
$$

Figure 6 illustrates the upper bound of Proposition 2 for $M\left(F_{2,4}\right)$.

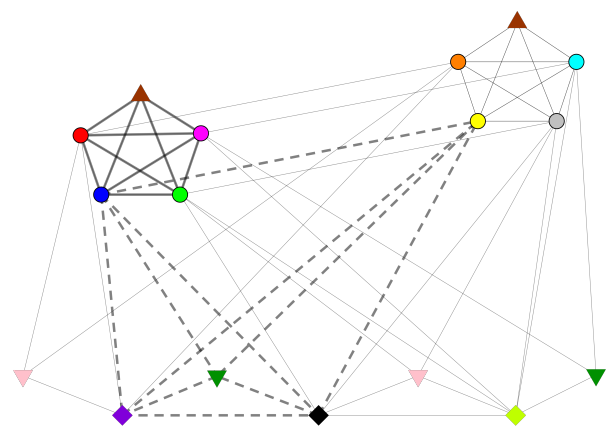

Figure 6: $r$-dynamic proper 13-coloring of $M\left(F_{2,4}\right)$, for all $r \geq 8$.

Now, we focus on determining the exact value of $\chi_{r}\left(M\left(F_{1, n}\right)\right)$. The following result, which establishes the exact value of $\chi_{n-1}\left(M\left(F_{m, n}\right)\right)$, whenever $n \geq m+2$, is useful to this end. 
Proposition 3. Let $m$ and $n$ be two positive integers such that $n \geq m+2$. Then,

$$
\chi_{n+1}\left(M\left(F_{m, n}\right)\right)=n+2 .
$$

Proof. From Lemma 1, we have that $n+2 \leq \chi_{n+1}\left(M\left(F_{m, n}\right)\right)$. In order to prove that this lower bound is reached, let $c$ be the proper $(n+2)$-coloring of the middle graph $M\left(F_{m, n}\right)$ that is defined so that, for each pair of non-negative integers $i<m$ and $j<n$, we have that

$c\left(u_{i}\right)=n, \quad c\left(v_{j}\right)= \begin{cases}(j-1) \bmod n, & \text { if }\left\{\begin{array}{l}n \text { is even, } \\ n \text { is odd and } j<n-1,\end{array}\right. \\ n+1, & \text { otherwise. }\end{cases}$

and

$$
c\left(u_{i} v_{j}\right)=(i+j) \bmod n,
$$

and, for each non-negative integer $k<n-1$, we have that

$$
c\left(v_{k} v_{k+1}\right)=n+1-(k \bmod 2) .
$$

Condition (1) holds and hence, $\chi_{n+1}\left(M\left(F_{m, n}\right)\right)=n+2$.

Figure 7 illustrates Proposition 3, for $(m, n) \in\{(1,3),(2,4)\}$.
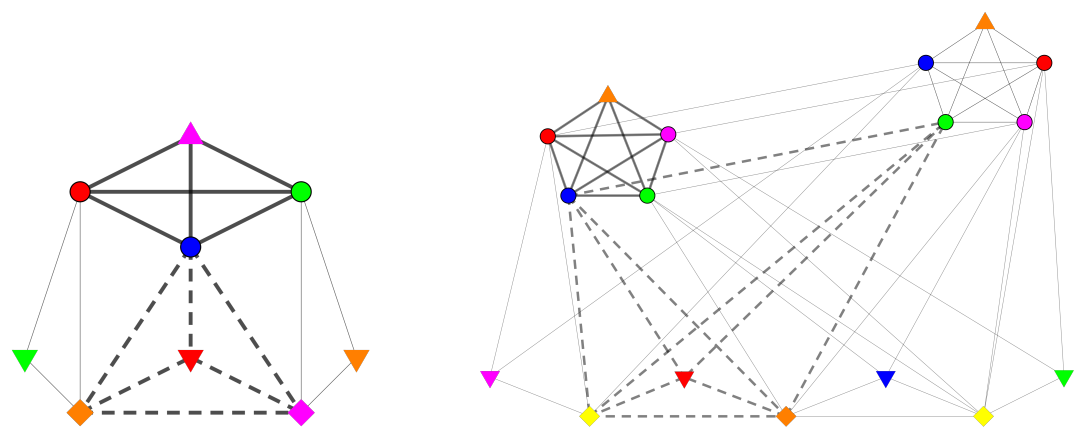

Figure 7: $(n+1)$-dynamic proper $(n+2)$-coloring of the middle graph $M\left(F_{m, n}\right)$, for $(m, n) \in\{(1,3),(2,4)\}$.

Let us finish this section by determining the exact value of $\chi_{r}\left(M\left(F_{1, n}\right)\right)$. 
Theorem 1. Let $n>2$ and $r$ be two positive integers. Then,

$$
\chi_{r}\left(M\left(F_{1, n}\right)\right)= \begin{cases}8, & \text { if } n=3 \text { and } r \geq 6, \\ n+1, & \text { if } r \leq n, \\ n+2, & \text { if } r=n+1, \\ n+4, & \text { if } r=n+2, \\ n+6, & \text { otherwise. }\end{cases}
$$

Proof. The case $r \notin\{n+1, n+2\}$ follows from Proposition 2. In addition, the case $r=n+1$ holds from Proposition 3. So, let us focus on the case $r=n+2$. To this end, let us suppose the existence of an $(n+2)$-dynamic proper $(n+3)$-coloring $c$ of the middle graph $M\left(F_{1, n}\right)$.

From Condition (1), we have that the set

$$
c\left(V\left(K_{M\left(F_{m, n}\right)}(0)\right)\right) \cup\left\{c\left(v_{1}\right), c\left(v_{0} v_{1}\right)\right\}
$$

is formed by $n+3$ distinct colors. Then, since $d\left(u_{0} v_{0}\right)=n+2$ and $v_{0} \in$ $N\left(u_{0} v_{0}\right)$, it must be $c\left(v_{0}\right) \in\left\{c\left(v_{1}\right), c\left(v_{1} v_{2}\right)\right\}$. But then, $\mid c\left(N\left(v_{0} v_{1}\right) \mid<d\left(v_{0} v_{1}\right)=\right.$ $5 \leq r$, which contradicts Condition (1). Hence, $n+4 \leq \chi_{r}\left(M\left(F_{1, n}\right)\right)$.

In order to prove that the previous lower bound is indeed reached, it is enough to consider the $(n+2)$-dynamic proper $(n+4)$-coloring $c$ of the middle graph $M\left(F_{m, n}\right)$ that is defined so that $c\left(u_{0}\right)=n$, and for each pair of nonnegative integers $j<n$ and $k<n+1$, we have that

$$
c\left(v_{j}\right)=\left\{\begin{array}{ll}
n+3, & \text { if } j \text { is even, } \\
n, & \text { otherwise. }
\end{array} \quad c\left(u_{0} v_{j}\right)=j \bmod n,\right.
$$

and $c\left(v_{k} v_{k+1}\right)=n+1+(k \bmod 2)$.

Figure 8 illustrates Theorem 1 for $(n, r) \in\{(3,5),(3,6)\}$.
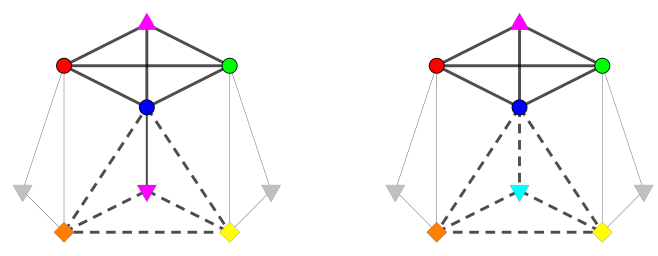

Figure 8: $r$-dynamic proper $\ell$-coloring of the middle graph $M\left(F_{1,3}\right)$, for $(r, \ell)=$ $(5,7)$ (left) and $(r, \ell)=(6,8)$ (right). 


\section{Dynamic coloring of the total graph of a fan graph}

This section deals with the $r$-dynamic chromatic number of the total graph $T\left(F_{m, n}\right)$. Notice that $V\left(T\left(F_{m, n}\right)\right)=V\left(M\left(F_{m, n}\right)\right)$ and

$$
E\left(T\left(F_{m, n}\right)\right)=E\left(M\left(F_{m, n}\right)\right) \cup E\left(F_{m, n}\right) .
$$

Thus, the vertex degrees of the total graph $T\left(F_{m, n}\right)$ coincide with those ones of the middle graph $M\left(F_{m, n}\right)$, except for

$d\left(u_{i}\right)=2 n \quad$ and $\quad d\left(v_{j}\right)= \begin{cases}2 m+2, & \text { if } j \in\{0, n-1\}, \\ 2 m+4, & \text { otherwise. }\end{cases}$

for every pair of non-negative integers $i<m$ and $j<n$. Hence, $\delta\left(T\left(F_{m, n}\right)\right)=$ $\min \{2 m+2,2 n\}$ and $\Delta\left(T\left(F_{m, n}\right)\right)=\max \{2 m+4,2 n\}$. Let us establish a pair of sharp bounds for the $r$-dynamic chromatic number $\chi_{r}\left(T\left(F_{m, n}\right)\right)$.

Proposition 4. Let $m, n$ and $r$ be three positive integers such that $n>2$. Then,

$\max \{m+3, n+1\} \leq \chi_{r}\left(T\left(F_{m, n}\right)\right) \leq\left\{\begin{array}{ll}m \cdot(n+1)+5, & \text { if } n \in\{3,4\}, \\ m \cdot(n+1)+n, & \text { otherwise. }\end{array}\right.$.

The lower bound is reached for every positive integer $r<\max \{m+3, n+1\}$, whereas the upper bound is reached for every positive integer $r \geq \Delta\left(T\left(F_{m, n}\right)\right)$.

Proof. Since the middle graph $M\left(F_{m, n}\right)$ is a spanning subgraph of the total graph $T\left(F_{m, n}\right)$, both the $(n+1)$-clique $K_{M\left(F_{m, n}\right)}(0)$ and the $(m+3)$-clique $K_{M\left(F_{m, n}\right)}^{\prime}(1)$ described in Section 3 are subgraphs of $T\left(F_{m, n}\right)$. Due to it, the result concerning the lower bound follows similarly to the proof of Proposition 2. Furthermore, in order to deal with the upper bound, Lemma 1 enables us to focus on the case $r=\Delta\left(T\left(F_{m, n}\right)\right)$. Under such assumption, Condition (1) implies that no two vertices in $V\left(T\left(F_{m, n}\right)\right) \backslash\left\{v_{k} v_{k+1}: 0 \leq k<n-1\right\}$ are equally colored. Hence, $(m+1) \cdot n+m \leq \chi_{r}\left(T\left(F_{m, n}\right)\right)$. In order to prove that this lower bound is reached whenever $n>4$, let us consider the proper coloring $c$ of the total graph $T\left(F_{m, n}\right)$ that is defined so that, for every pair of non-negative integers $i<m$ and $j<n$, we have that

$$
c\left(u_{i}\right)=m \cdot n+i, \quad c\left(v_{j}\right)=m \cdot(n+1)+j,
$$

and $c\left(u_{i} v_{j}\right)=(i+j) \bmod n$, and, for each non-negative integer $k<n-1$, we have that

$$
c\left(v_{k} v_{k+1}\right)=m \cdot(n+1)+((k+3) \bmod n) .
$$




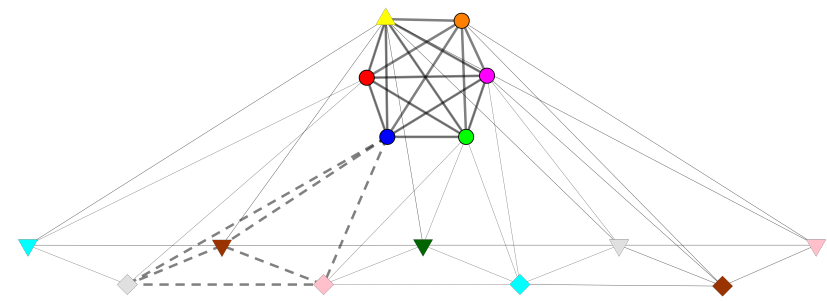

Figure 9: $r$-dynamic proper 11-coloring of $T\left(F_{1,5}\right)$, for all $r \geq 10$.

Condition (1) holds and hence, $\chi_{\Delta\left(T\left(F_{m, n}\right)\right)}\left(T\left(F_{m, n}\right)\right)=(m+1) \cdot n+m$. Figure 9 illustrates this upper bound for the total graph $T\left(F_{1,5}\right)$.

The previous lower bound is, however, not reached for $n \in\{3,4\}$. Thus, for $n=3$, it is simply verified that no two vertices in the total graph $T\left(F_{m, 3}\right)$ may equally be colored. Hence,

$$
\chi_{\Delta\left(T\left(F_{m, 3}\right)\right)}\left(T\left(F_{m, 3}\right)\right)=\left|V\left(T\left(F_{m, 3}\right)\right)\right|=4 m+5 .
$$

Further, for $n=4$, it is straightforwardly verified that the vertex $v_{1} v_{2}$ requires an extra color and hence, $5 m+5 \leq \chi_{\Delta\left(T\left(F_{m, 4}\right)\right)}\left(T\left(F_{m, 4}\right)\right)$. In order to prove that this lower bound is reached, it is enough to consider the same proper coloring $c$ that we have described for the case $n>4$, except for $c\left(v_{0} v_{1}\right)=$ $5 m+3, c\left(v_{1} v_{2}\right)=5 m+4$ and $c\left(v_{2} v_{3}\right)=5 m$. Figure 10 illustrates this upper bound for the total graph $T\left(F_{2,4}\right)$.

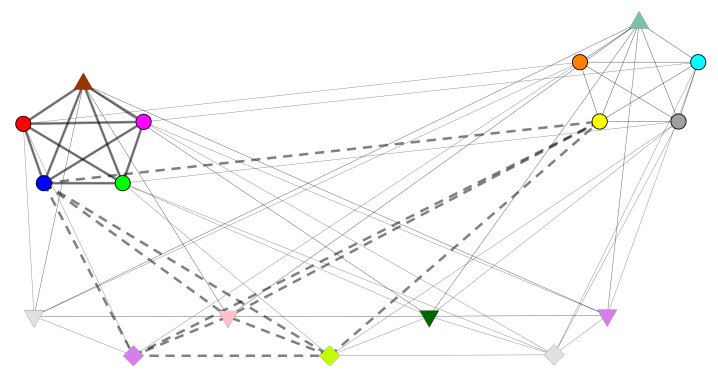

Figure 10: $r$-dynamic proper 15-coloring of $T\left(F_{2,4}\right)$, for all $r \geq 8$.

The following result establishes the exact value of $\chi_{r}\left(T\left(F_{1, n}\right)\right)$. 
Theorem 2. Let $n>2$ and $r$ be two positive integers. Then,

$$
\chi_{r}\left(T\left(F_{1, n}\right)\right)= \begin{cases}8, & \text { if }(n, r)=(3,5), \\ 9, & \text { if } n=3 \text { and } r \geq 6, \\ 10, & \text { if } n=4 \text { and } r \geq 8, \\ n+1, & \text { if } r \leq n, \\ n+3, & \text { if } r=n+1, \\ n+4, & \text { if } r=n+2 \geq 6, \\ n+6, & \text { if } n+3 \leq r<\min \{n+5,2 n\}, \\ r+1, & \text { if } n+5 \leq r<2 n, \\ 2 n+1, & \text { otherwise. }\end{cases}
$$

Proof. Proposition 4 implies both cases $r \leq n$ and $r \geq 2 n$. So, let us focus on the case $r=n+\rho$, for each positive integer $\rho<n$. To this end, let $c$ be an $(n+\rho)$-dynamic proper $\chi_{n+\rho}\left(T\left(F_{1, n}\right)\right)$-coloring of the total graph $T\left(F_{1, n}\right)$. Without loss of generality, since the $(n+1)$-clique $K_{M\left(F_{m, n}\right)}(0)$ is a subgraph of this total graph, we may assume that $c\left(u_{0}\right)=n$ and $c\left(u_{0} v_{j}\right)=j$, for all $j<n$. The following study of cases arises.

- Case $\rho=1$.

From Lemma 1, we have that $n+2 \leq \chi_{n+1}\left(T\left(F_{1, n}\right)\right)$. Let us prove that the assumption that this lower bound is reached gives rise to a contradiction. Since $n+1 \leq \min \left\{d\left(u_{0}\right), d\left(u_{0} v_{j}\right): j<n\right\}$, Condition (1), together with the just imposed assumptions on the map $c$, implies that the color $n+1$ belongs to the set

$$
\begin{aligned}
\left\{c\left(v_{j}\right): j<n\right\} & \cap\left\{c\left(v_{0}\right), c\left(v_{0} v_{1}\right)\right\} \\
& \cap \bigcap_{k=1}^{n-2}\left\{c\left(v_{k-1} v_{k}\right), c\left(v_{k}\right), c\left(v_{k} v_{k+1}\right)\right\} \\
& \cap\left\{c\left(v_{n-2} v_{n-1}\right), c\left(v_{n-1}\right)\right\} .
\end{aligned}
$$

The following three subcases arise:

- Subcase $n=3$. From (2) and the fact that the map $c$ is a proper coloring, we have that either $c\left(v_{0}\right)=c\left(v_{1} v_{2}\right)=4$ or $c\left(v_{0} v_{1}\right)=$ $c\left(v_{2}\right)=4$. In both cases, any coloring of the vertex $v_{1}$ contradicts either Condition (1) or the fact that the map $c$ is a proper coloring.

- Subcase $n=4$. From (2), together with the fact that the map $c$ is a proper coloring, implies that either $c\left(v_{0}\right)=c\left(v_{1} v_{2}\right)=c\left(v_{3}\right)=5$ or $c\left(v_{0} v_{1}\right)=c\left(v_{2}\right)=5$. The first option implies that $\left|c\left(N\left(v_{0} v_{1}\right)\right)\right|<$ $5=d\left(v_{0} v_{1}\right)$, which contradicts Condition (1). 
So, let us assume the second option. Since $d\left(v_{0}\right)=4$ and $d\left(v_{0} v_{1}\right)=$ 5 , Condition (1), together with the already colored vertices, implies that $\left\{c\left(v_{0}\right), c\left(v_{1}\right)\right\}=\{2,3\}$ and $c\left(v_{1} v_{2}\right)=4$. But then, $\left|c\left(N\left(v_{1}\right)\right)\right|=4$, which contradicts Condition (1), because $d\left(v_{1}\right)=6$.

- Subcase $n \geq 5$. Here, (2) contradicts Condition (1), because it would imply the existence of a non-negative integer $k<n-1$ such that $\left|c\left(N\left(v_{k} v_{k+1}\right)\right)\right|<d\left(v_{k} v_{k}+1\right) \leq 6 \leq n+1$.

Thus, $n+3 \leq \chi_{r}\left(T\left(F_{1, n}\right)\right)$. In order to prove that this bound is reached, let the map $c$ be defined so that

$$
c\left(v_{j}\right)=n+1+(j \bmod 2) \quad \text { and } \quad c\left(v_{k} v_{k+1}\right)=(k+2) \bmod n,
$$

for all non-negative integers $j<n$ and $k<n-1$. Condition (1) holds and hence, $\chi_{n+1}\left(T\left(F_{1, n}\right)\right)=n+3$. Figure 11 illustrates the case $n \in\{3,4\}$.
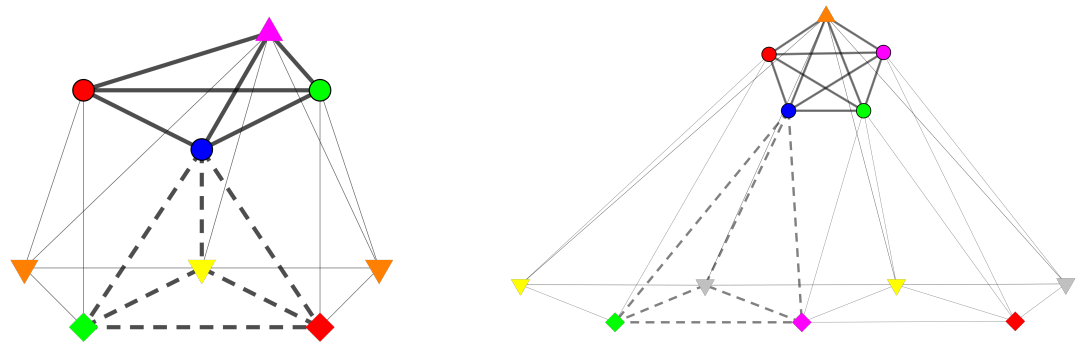

Figure 11: $(n+1)$-dynamic proper $(n+3)$-coloring of $T\left(F_{1, n}\right)$, for $n \in\{3,4\}$.

- Case $\rho=2$.

Lemma 1 implies that $n+3 \leq \chi_{n+2}\left(T\left(F_{1, n}\right)\right)$. If this lower bound were reached, then, similarly to the previous case, both colors $n+1$ and $n+2$ should be contained within each one of the sets that we have described in (2). In particular, since the map $c$ is a proper coloring, it should be $c\left(v_{0}\right)=c\left(v_{1} v_{2}\right)$. It would imply that $\left|c\left(N\left(v_{0} v_{1}\right)\right)\right|<5=d\left(v_{0} v_{1}\right)$, which contradicts Condition (1). Hence, $n+4 \leq \chi_{n+2}\left(T\left(F_{1, n}\right)\right)$. This lower bound is not reached for $n=3$, which requires an eight color. Otherwise, Condition (1) would imply that $\left\{c\left(v_{0}\right), c\left(v_{0} v_{1}\right), c\left(v_{1} v_{2}\right)\right\}=\{4,5,6\}$ and $c\left(v_{0}\right)=c\left(v_{2}\right)$. But then, any coloring of the vertex $v_{1}$ would contradict either Condition (1) or the fact that the map $c$ is a proper coloring. Figure 12 (left) illustrates that $\chi_{5}\left(T\left(F_{1,3}\right)\right)=8$. 

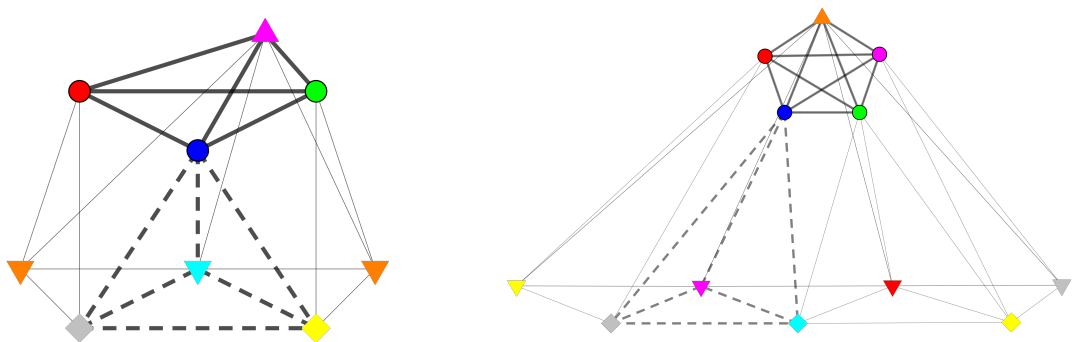

Figure 12: $(n+2)$-dynamic proper 8-coloring of $T\left(F_{1, n}\right)$, for $n \in\{3,4\}$.

Now, in order to prove that the lower bound $n+4$ is reached for all $n \geq 4$, let the map $c$ be defined so that

$c\left(v_{0}\right)=n+1, \quad c\left(v_{j}\right)=(j+2) \bmod n, \quad c\left(v_{n-1}\right)=n+1+(n \bmod 3)$, and $c\left(v_{k} v_{k+1}\right)=n+1+((k+1) \bmod 3)$, for all positive integer $j<n-1$ and all non-negative integer $k<n-1$. Condition (1) holds and hence, $\chi_{n+2}\left(T\left(F_{1, n}\right)\right)=n+4$, for all $n \geq 4$. Figure 12 (right) illustrates the case $n=4$.

- Case $3 \leq \rho<\min \{5, n\}$.

If $r \geq n+3$, then Condition (1) implies that no two distinct vertices within the neighborhood of any given vertex $v \in V\left(T\left(F_{1, n}\right)\right) \backslash\left\{u_{0}\right\}$ are equally colored. Particularly, if we focus our attention on the vertex $v_{1}$, then it is simply verified that $n+6 \leq \chi_{r}\left(T\left(F_{1, n}\right)\right)$, for every positive integer $r \geq n+3$. In order to prove that this lower bound is reached, let the map $c$ be defined so that

$c\left(v_{j}\right)=n+1+(2 j \bmod 5) \quad$ and $\quad c\left(v_{k} v_{k+1}\right)=n+1+((2 k+1) \bmod 5)$,

for all non-negative integers $j<n$ and $k<n-1$. Condition (1) holds and hence, $\chi_{r}\left(T\left(F_{1, n}\right)\right)=n+6$, whenever $n+3 \leq r \leq \min \{n+5,2 n\}$. Figure 13 illustrates the case $n \in\{3,4\}$.

- Case $5 \leq \rho<n$.

From Lemma 1 , we have that $n+\rho+1 \leq \chi_{n+\rho}\left(T\left(F_{1, n}\right)\right)$. In order to prove that this lower bound is reached, it is enough to consider the map $c$ that we have just described in the previous case, except for $c\left(v_{i}\right)=n+6+i$, for all non-negative integer $i<\rho-5$. 

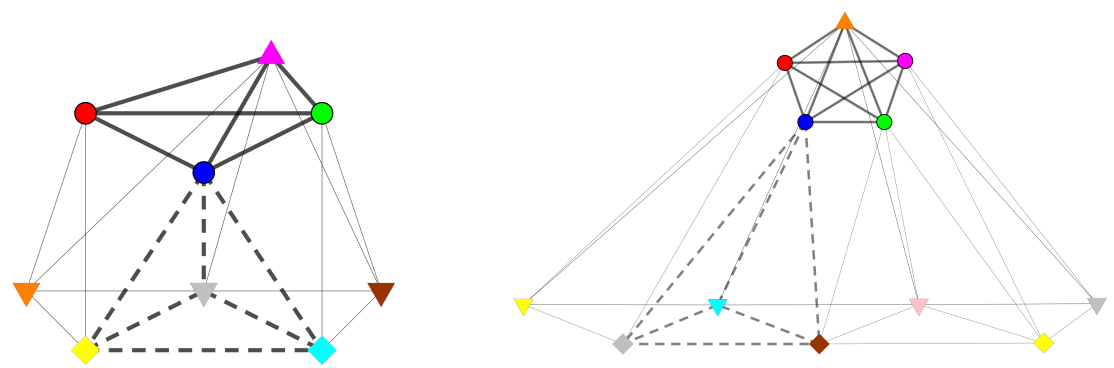

Figure 13: $r$-dynamic proper $\ell$-coloring of the total graph $T\left(F_{1, n}\right)$, for all $r \geq 6$, with $(n, \ell) \in\{(3,9),(4,10)\}$.

\section{Dynamic coloring of the central graph of a fan graph}

Let us focus now on the $r$-dynamic chromatic number of the central graph $C\left(F_{m, n}\right)$. Its set of vertices is

$$
\begin{aligned}
V\left(C\left(F_{m, n}\right)\right)= & \left\{u_{0}, \ldots, u_{m-1}, v_{0}, \ldots, v_{n-1}, w_{0,0}, \ldots, w_{0, n-1}, \ldots, w_{m-1,0}, \ldots,\right. \\
& \left.w_{m-1, n-1}, w_{0,1}^{\prime}, \ldots, w_{n-2, n-1}^{\prime}\right\} .
\end{aligned}
$$

Here, for each pair of non-negative integers $i<m$ and $j<n$, the vertex $w_{i, j}$ is the third one within the path of size two joining both vertices $u_{i}$ and $v_{j}$. Similarly, for each non-negative integer $k<n-1$, the vertex $w_{k, k+1}^{\prime}$ is the third one within the path of size two joining both vertices $v_{k}$ and $v_{k+1}$. In particular,

$$
d\left(u_{i}\right)=d\left(v_{j}\right)=m+n-1 \quad \text { and } \quad d\left(w_{i, j}\right)=d\left(w_{k, k+1}^{\prime}\right)=2
$$

for all non-negative integers $i<m, j<n$ and $k<n-1$. Hence, $\delta\left(C\left(F_{m, n}\right)\right)=$ 2 and $\Delta\left(C\left(F_{m, n}\right)\right)=m+n-1$.

Let $K_{C\left(F_{m, n}\right)}$ and $K_{C\left(F_{m, n}\right)}^{\prime}$ denote, respectively, the $m$ - and $\left\lceil\frac{n}{2}\right\rceil$-cliques within the central graph $C\left(F_{m, n}\right)$ that are described by the set of vertices

$$
V\left(K_{C\left(F_{m, n}\right)}\right)=\left\{u_{0}, \ldots, u_{m-1}\right\} \text { and } V\left(K_{C\left(F_{m, n}\right)}^{\prime}\right)=\left\{v_{2 i}: 0 \leq i<\left\lceil\frac{n}{2}\right\rceil\right\} .
$$

From now on, and similarly to the already studied families of graphs, every dynamic coloring of the central graph $C\left(F_{m, n}\right)$ is illustrated so that the edges of the just described $m$ - and $\left\lceil\frac{n}{2}\right\rceil$-cliques are respectively highlighted with bold and dashed lines. In addition, the vertices of the sets $\left\{u_{0}, \ldots, u_{m-1}\right\}$, 
$\left\{v_{0}, \ldots, v_{n-1}\right\},\left\{w_{0,0}, \ldots, w_{m-1, n-1}\right\}$ and $\left\{w_{0,1}^{\prime}, \ldots, w_{n-2, n-1}^{\prime}\right\}$ are respectively distinguished by means of the symbols $\boldsymbol{\Lambda}, \boldsymbol{\nabla}, \bullet$ and $\bullet$.

The following result establishes the chromatic number of the central graph $C\left(F_{m, n}\right)$.

Proposition 5. Let $m$ and $n>2$ be two positive integers. Then,

$$
\chi\left(C\left(F_{m, n}\right)\right)= \begin{cases}3, & \text { if } m \leq\left\lceil\frac{n}{2}\right\rceil=2, \\ \left\lceil\frac{n}{2}\right\rceil, & \text { if } m \leq\left\lceil\frac{n}{2}\right\rceil \neq 2, \\ m, & \text { otherwise. }\end{cases}
$$

Proof. Let us study each case separately.

- Case $m \leq\left\lceil\frac{n}{2}\right\rceil=2$.

Since the cycle $C_{5}$ described by the subset of vertices

$$
\left\{v_{0}, v_{0} v_{1}, v_{1}, v_{1} v_{2}, v_{2}\right\} \subset V\left(C\left(F_{m, n}\right)\right)
$$

is an induced subgraph of the central graph $C\left(F_{m, n}\right)$, Lemma 2 implies that $3 \leq \chi\left(C\left(F_{m, n}\right)\right)$. This lower bound is sharp for all $m \leq\left\lceil\frac{n}{2}\right\rceil=2$. To see it, it is enough to define a proper 3-coloring $c$ of the central graph $C\left(F_{m, n}\right)$ so that

$$
c\left(u_{i}\right)=i, \quad c\left(w_{i, j}\right)=2, \quad c\left(v_{j}\right)=\left\lfloor\frac{j}{2}\right\rfloor \quad \text { and } \quad c\left(w_{k, k+1}^{\prime}\right)=2,
$$

for all non-negative integers $i<m, j<n$ and $k<n-1$. Condition (1) holds and hence, $\chi\left(C\left(F_{m, n}\right)\right)=3$, for all $m \leq\left\lceil\frac{n}{2}\right\rceil=2$. Figure 14 (left) illustrates the central graph $C\left(F_{2,4}\right)$.
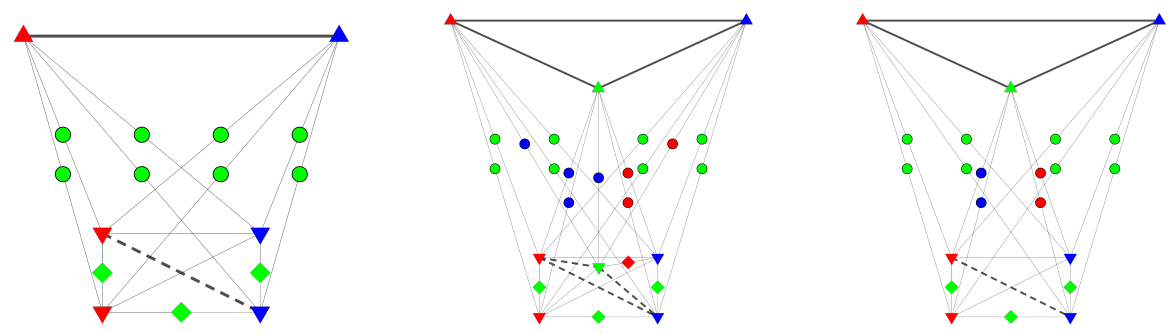

Figure 14: Proper 3-colorings of $C\left(F_{2,4}\right), C\left(F_{3,5}\right)$ and $C\left(F_{3,4}\right)$. 
- Case $m \leq\left\lceil\frac{n}{2}\right\rceil \neq 2$.

The existence of the $\left\lceil\frac{n}{2}\right\rceil$-clique $K_{C\left(F_{m, n}\right)}^{\prime}$ as a subgraph of the central graph $C\left(F_{m, n}\right)$, together with Lemma 2 implies that $\left\lceil\frac{n}{2}\right\rceil \leq \chi\left(C\left(F_{m, n}\right)\right)$. In order to prove that this lower bound is reached, we define a proper $\left\lceil\frac{n}{2}\right\rceil$-coloring $c$ of the central graph $C\left(F_{m, n}\right)$ so that

$$
\begin{aligned}
& c\left(w_{i, j}\right)= \begin{cases}\left\lceil\frac{n}{2}\right\rceil-1, & \text { if } \max \left\{i,\left\lceil\frac{j}{2}\right\rceil\right\}<\left\lceil\frac{n}{2}\right\rceil-1, \\
(i+1) \bmod \left\lfloor\frac{n}{2}\right\rfloor, & \text { if } i \leq\left\lceil\frac{n}{2}\right\rceil-1=j, \\
\left(\left\lfloor\frac{j}{2}\right\rfloor+1\right) \bmod \left\lfloor\frac{n}{2}\right\rfloor, & \text { otherwise. }\end{cases} \\
& c\left(u_{i}\right)=i, \quad c\left(v_{j}\right)=\left\lfloor\frac{j}{2}\right\rfloor \quad \text { and } \quad c\left(w_{k, k+1}^{\prime}\right)=\left\lceil\frac{n}{2}\right\rceil-1,
\end{aligned}
$$

for all non-negative integers $i<m, j<n$ and $k<n-1$. Condition (1) holds and hence, $\chi\left(C\left(F_{m, n}\right)\right)=\left\lceil\frac{n}{2}\right\rceil$, for all $m \leq\left\lceil\frac{n}{2}\right\rceil \neq 2$. Figure 14 (center) illustrates the central graph $C\left(F_{3,5}\right)$.

- Case $m>\left\lceil\frac{n}{2}\right\rceil$.

The existence of the $m$-clique $K_{C\left(F_{m, n}\right)}$ as a subgraph of the central graph $C\left(F_{m, n}\right)$, together with Lemma 2 implies that $m \leq \chi\left(C\left(F_{m, n}\right)\right)$. In order to prove that this lower bound is reached, we define a proper $m$-coloring $c$ of the central graph $C\left(F_{m, n}\right)$ so that

$$
\begin{array}{cl}
c\left(u_{i}\right)=i, & c\left(w_{i, j}\right)= \begin{cases}m-1, & \text { if } i<m-1, \\
\left(\left\lfloor\frac{j}{2}\right\rfloor+1\right) \bmod \left\lfloor\frac{n}{2}\right\rfloor, & \text { otherwise. }\end{cases} \\
c\left(v_{j}\right)=\left\lfloor\frac{j}{2}\right\rfloor \quad \text { and } \quad c\left(w_{k, k+1}^{\prime}\right)=m-1,
\end{array}
$$

for all non-negative integers $i<m, j<n$ and $k<n-1$. Condition (1) holds and hence, $\chi\left(C\left(F_{m, n}\right)\right)=m$, for all $m>\left\lceil\frac{n}{2}\right\rceil$. Figure 14 (right) illustrates the central graph $C\left(F_{3,4}\right)$.

The following result describes a pair of sharp bounds for the case $r>1$.

Proposition 6. Let $m, n$ and $r$ be three positive integers such that $n>2$ and $r>1$. Then,

$$
m+n \leq \chi_{r}\left(C\left(F_{m, n}\right)\right) \leq \begin{cases}n+2, & \text { if } m=1, \\ 2 m+n-1, & \text { if } m>1 .\end{cases}
$$


The lower bound is reached whenever $r<\max \{m, n\}$, whereas the upper bound is reached whenever $r \geq \Delta\left(C\left(F_{m, n}\right)\right)$.

Proof. Throughout the proof, let $c$ denote an $r$-dynamic proper $\chi_{r}\left(C\left(F_{m, n}\right)\right)$ coloring of the central graph $C\left(F_{m, n}\right)$. Since $d\left(w_{i, j}\right)=d\left(w_{k, k+1}^{\prime}\right)=2$, for every non-negative integers $i<m, j<n$ and

$$
\left\{c\left(u_{0}\right), \ldots, c\left(u_{m-1}\right), c\left(v_{0}\right), \ldots, c\left(v_{n-1}\right)\right\}
$$

is formed by $m+n$ distinct colors. Hence, $m+n \leq \chi_{r}\left(C\left(F_{m, n}\right)\right)$. In order to prove that this lower bound is reached whenever $r<\max \{m, n\}$, the following two cases arise.

- Case $m \leq n$.

Let the map $c$ be defined so that

$$
\begin{array}{lrr}
c\left(u_{i}\right)=i, & c\left(w_{i, j}\right)=m+((j+1) \bmod n), \\
c\left(v_{j}\right)=m+j & \text { and } \quad c\left(w_{k, k+1}^{\prime}\right)=0,
\end{array}
$$

for all non-negative integers $i<m, j<n$ and $k<n-1$. Condition (1) holds and hence, $\chi_{r}\left(C\left(F_{m, n}\right)\right)=m+n$. Figure 15 (left) illustrates the case $(m, n)=(1,4)$.
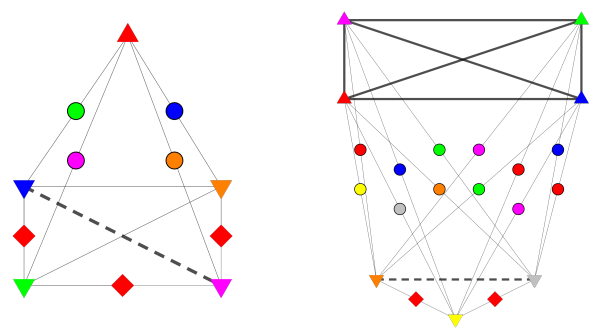

Figure 15: $r$-dynamic proper colorings of the central graphs $C\left(F_{1,4}\right)$ and $C\left(F_{4,3}\right)$, for all $r \in\{2,3\}$.

- Case $n<m$.

Let the map $c$ be defined so that

$$
c\left(u_{i}\right)=i, \quad c\left(w_{i, j}\right)= \begin{cases}m+((j+1) \bmod n), & \text { if } i=0, \\ (i+j+1) \bmod m, & \text { otherwise. }\end{cases}
$$




$$
c\left(v_{j}\right)=m+j \quad \text { and } \quad c\left(w_{k, k+1}^{\prime}\right)=0,
$$

for all non-negative integers $i<m, j<n$ and $k<n-1$. Condition (1) holds and hence, $\chi_{r}\left(C\left(F_{m, n}\right)\right)=m+n$. Figure 15 (right) illustrates the case $(m, n)=(4,3)$.

Now, in order to deal with the upper bound, Lemma 1 enables us to focus on the case $r=\Delta\left(C\left(F_{m, n}\right)\right)$. The following two cases arise.

- Case $m=1$.

Condition (1) implies that the set $\left\{c\left(w_{0,1}^{\prime}\right), \ldots, c\left(w_{n-2, n-1}^{\prime}\right)\right\}$ is formed by, at least, two distinct colors. Since one of them may coincide with $c\left(u_{0}\right)$, and none of them belongs to the set of $n$ distinct colors $\left\{c\left(v_{0}\right), \ldots\right.$, $\left.c\left(v_{n-1}\right)\right\}$, we have that $n+2 \leq \chi_{\Delta\left(C\left(F_{m, n}\right)\right)}\left(C\left(F_{m, n}\right)\right)$. In order to prove that this lower bound is reached, let the map $c$ be defined so that

$$
c\left(u_{0}\right)=0, \quad c\left(w_{0, j}\right)=j+2, \quad c\left(v_{j}\right)=j+1
$$

and

$$
c\left(w_{k, k+1}^{\prime}\right)= \begin{cases}0, & \text { if } k \equiv n(\bmod 2), \\ n+1, & \text { if } k \not \equiv n(\bmod 2) .\end{cases}
$$

for every pair of non-negative integers $j<n$ and $k<n-1$. Condition (1) holds and hence, $\chi_{\Delta\left(C\left(F_{m, n}\right)\right)}\left(C\left(F_{1, n}\right)\right)=n+2$. Figure 16 (left) illustrates the central graph $C\left(F_{1,4}\right)$.
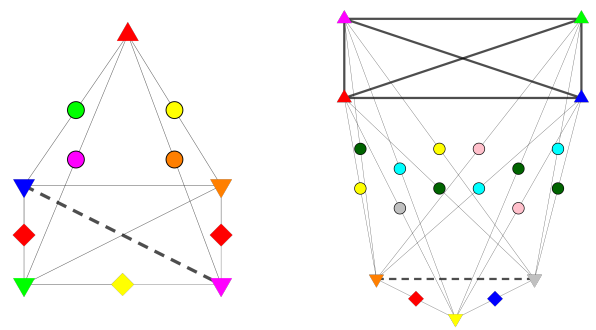

Figure 16: $r$-dynamic proper colorings of the central graphs $C\left(F_{m, n}\right)$, for all $r \geq m+n-1$, with $(m, n) \in\{(1,4),(4,3)\}$.

- Case $m>1$.

For each non-negative integer $j<n$, since $\left|c\left(N\left(v_{j}\right)\right)\right|=d\left(v_{j}\right)=m+n-1$, Condition (1) implies that the set $\left\{c\left(w_{0, j}\right), \ldots, c\left(w_{m-1, j}\right)\right\}$ is formed by $m$ distinct colors, none of them in the set

$$
\left\{c\left(u_{0}\right), \ldots, c\left(u_{m-1}\right), c\left(v_{0}\right), \ldots, c\left(v_{n-1}\right)\right\}
$$


except for

$$
\begin{cases}\left\{c\left(v_{1}\right)\right\}, & \text { if } j=0, \\ \left\{c\left(v_{j-1}\right), c\left(v_{j+1}\right)\right\}, & \text { if } 0<j<n-1, \\ \left\{c\left(v_{n-2}\right)\right\}, & \text { if } j=n-1,\end{cases}
$$

As a consequence, $2 m+n-1 \leq \chi_{\Delta\left(C\left(F_{m, n}\right)\right)}\left(C\left(F_{m, n}\right)\right)$. In order to prove that this lower bound is reached, let the map $c$ be defined so that

$$
\begin{gathered}
c\left(w_{i, j}\right)= \\
\begin{cases}m+j+1, & \text { if } i=0 \text { and } j<n-1, \\
2 m+n-2, & \text { if }(i, j)=(0, n-1), \\
m+n-2, & \text { if }(i, j)=(m-1, n-1), \\
m+n+((i+j-1) \bmod (m-1)), & \text { otherwise. }\end{cases} \\
c\left(u_{i}\right)=i, \quad c\left(v_{j}\right)=m+j \quad \text { and } \quad c\left(w_{k, k+1}^{\prime}\right)= \begin{cases}0, & \text { if } k \text { is even, } \\
1, & \text { if } k \text { is odd } .\end{cases}
\end{gathered}
$$

for all non-negative integers $i<m, j<n$ and $k<n-1$. Condition (1) holds and hence, $\chi_{\Delta\left(C\left(F_{m, n}\right)\right)}\left(C\left(F_{1, n}\right)\right)=2 m+n-1$. Figure 16 (right) illustrates the case $(m, n)=(4,3)$.

As an immediate consequence of Propositions 5 and 6 , the following result establishes the $r$-dynamic chromatic number of the central graph $C\left(F_{1, n}\right)$.

Theorem 3. Let $n>2$ and $r$ be two positive integers. Then,

$$
\chi_{r}\left(C\left(F_{1, n}\right)\right)= \begin{cases}3, & \text { if } r=1 \text { and } n \in\{3,4\}, \\ \left\lceil\frac{n}{2}\right\rceil, & \text { if } r=1 \text { and } n>4, \\ n+1, & \text { if } 2 \leq r<n, \\ n+2, & \text { otherwise. }\end{cases}
$$

\section{Dynamic coloring of the line graph of a fan graph}

Let us finish this paper by dealing with the $r$-dynamic chromatic number of the line graph $L\left(F_{m, n}\right)$. Its set of vertices is $V\left(L\left(F_{m, n}\right)\right)=E\left(F_{m, n}\right)$ and its set of edges is 


$$
\begin{gathered}
E\left(L\left(F_{m, n}\right)\right)= \\
\left\{\left(u_{i} v_{j}\right)\left(u_{i} v_{k}\right),\left(u_{i} v_{j}\right)\left(v_{l} v_{j}\right): 0 \leq i, l<m, \text { and } 0 \leq j, k<n\right\} \\
\cup\left\{\left(u_{i} v_{j}\right)\left(v_{j-1} v_{j}\right),\left(u_{i} v_{j}\right)\left(v_{j} v_{j+1}\right): 0 \leq i<m \text { and } 0<j<n-1\right\} \\
\cup\left\{\left(u_{i} v_{0}\right)\left(v_{0} v_{1}\right),\left(u_{i} v_{n-1}\right)\left(v_{n-2} v_{n-1}\right): 0 \leq i<m\right\} .
\end{gathered}
$$

For all non-negative integers $i<m, j<n$ and $k<n-1$, we have that

$$
d\left(u_{i} v_{j}\right)= \begin{cases}m+n-1, & \text { if } j \in\{0, n-1\}, \\ m+n, & \text { otherwise. }\end{cases}
$$

and

$$
d\left(v_{k} v_{k+1}\right)= \begin{cases}2 m+1, & \text { if } k \in\{0, n-2\} \\ 2 m+2, & \text { otherwise }\end{cases}
$$

Hence,

$$
\delta\left(L\left(F_{m, n}\right)\right)= \begin{cases}2 m+1, & \text { if } m \leq n-2 \\ m+n-1, & \text { otherwise }\end{cases}
$$

and

$$
\Delta\left(L\left(F_{m, n}\right)\right)= \begin{cases}m+n, & \text { if } m \leq n-2, \\ 2 m+2, & \text { otherwise }\end{cases}
$$

Further, let $K_{L\left(F_{m, n}\right)}$ and $K_{L\left(F_{m, n}\right)}^{\prime}$ denote, respectively, the $n$ - and $(m+2)$ cliques within the line graph $L\left(F_{m, n}\right)$ that are described by the sets of vertices

$$
V\left(K_{L\left(F_{m, n}\right)}\right)=\left\{u_{0} v_{j}: 0 \leq j<n\right\}
$$

and

$$
V\left(K_{L\left(F_{m, n}\right)}^{\prime}\right)=\left\{v_{0} v_{1}, v_{1} v_{2}, u_{i} v_{1}: 0 \leq i<m\right\} .
$$

Again, similarly to the previous sections, every dynamic coloring of the line graph $L\left(F_{m, n}\right)$ is illustrated from now on so that the edges of the just described $n$ - and $(m+2)$-cliques are respectively highlighted with bold and dashed lines. Their corresponding vertices are respectively distinguished by means of the symbols $\boldsymbol{\Lambda}$ and $\mathbf{\nabla}$. The following result establishes a pair of sharp bounds for the $r$-dynamic chromatic number $\chi_{r}\left(L\left(F_{m, n}\right)\right)$.

Proposition 7. Let $m, n$ and $r$ be three positive integers such that $n>2$. Then,

$$
\max \{m+2, n\} \leq \chi_{r}\left(L\left(F_{m, n}\right)\right) \leq \begin{cases}m \cdot n+2, & \text { if } n=3 \\ m \cdot n+3, & \text { otherwise }\end{cases}
$$


The lower bound is reached whenever $r<\max \{m+2, n\}$, whereas the upper bound is reached whenever $r \geq \Delta\left(L\left(F_{m, n}\right)\right)$.

Proof. The lower bound holds straightforwardly from Lemma 2, once it is noticed, for instance, that both cliques $K_{L\left(F_{m, n}\right)}$ and $K_{L\left(F_{m, n}\right)}^{\prime}$ are subgraphs of the line graph $L\left(F_{m, n}\right)$. In order to prove that this lower bound is reached for every positive integer $r<\max \{m+2, n\}$, it is enough to consider the proper coloring $c$ of the line graph $L\left(F_{m, n}\right)$ such that

$$
c\left(u_{i} v_{j}\right)=(i+j) \bmod \max \{m+2, n\}
$$

and

$$
c\left(v_{k} v_{k+1}\right)=(m+k+1) \bmod \max \{m+2, n\},
$$

for all non-negative integers $i<m, j<n$ and $k<n-1$. Condition (1) holds and hence, $\chi_{r}\left(L\left(F_{m, n}\right)\right)=\max \{m+2, n\}$, whenever $r<\max \{m+2, n\}$. Figure 17 illustrates the case $(m, n) \in\{(2,3),(2,4)\}$.
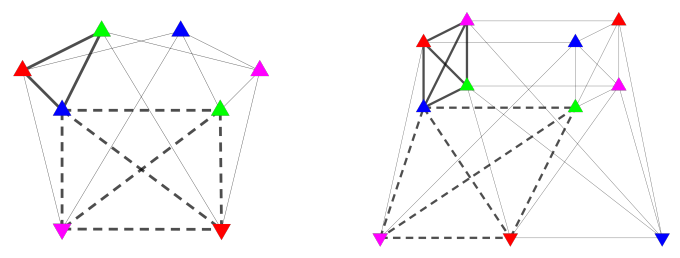

Figure 17: $r$-dynamic proper 4-colorings of the line graph $L\left(F_{m, n}\right)$, for all $r \leq \max \{m+2, n\}$, with $(m, n) \in\{(2,3),(2,4)\}$.

Now, in order to study the upper bound, Lemma 1 enables us to focus on the case $r=\Delta\left(L\left(F_{m, n}\right)\right)$. Let $c$ be a $\Delta\left(L\left(F_{m, n}\right)\right)$-dynamic proper $\chi_{\Delta\left(L\left(F_{m, n}\right)\right)}\left(\Delta\left(L\left(F_{m, n}\right)\right)\right)$-coloring of the line graph $L\left(F_{m, n}\right)$. In a similar way to the proof of the upper bound in Proposition 2, we may ensure that the set

$$
\left\{c\left(u_{0} v_{0}\right), \ldots, c\left(u_{m-1} v_{n-1}\right), c\left(v_{0} v_{1}\right), c\left(v_{1} v_{2}\right)\right\}
$$

is formed by $m \cdot n+2$ distinct colors. Moreover, if $n \geq 4$, then the vertex $v_{2} v_{3}$ requires an $(m \cdot n+3)^{\text {th }}$ extra color. Hence,

$$
\chi_{\Delta\left(L\left(F_{m, n}\right)\right)}\left(\Delta\left(L\left(F_{m, n}\right)\right)\right) \geq \begin{cases}3 m+2, & \text { if } n=3, \\ m \cdot n+3, & \text { otherwise. }\end{cases}
$$

In order to prove that this lower bound is reached, let the map $c$ be such that

$$
c\left(u_{i} v_{j}\right)=i \cdot n+j \quad \text { and } \quad c\left(v_{k} v_{k+1}\right)=m \cdot n+(k \bmod 3),
$$


for all positive integers $i<m, j<n$ and $k<n-1$. Condition (1) holds and hence, the mentioned lower bound is reached. Figure 18 illustrates the line graph $L\left(F_{2,4}\right)$.

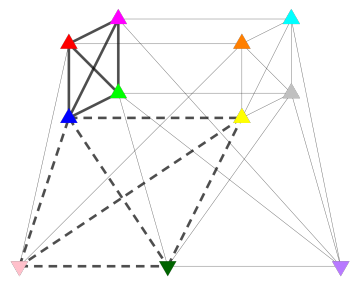

Figure 18: $r$-dynamic proper 11-coloring of $L\left(F_{2,4}\right)$, for all $r \geq 6$.

The following result establishes the $r$-dynamic chromatic number of the line graph $L\left(F_{1, n}\right)$.

Theorem 4. Let $n>2$ and $r$ be two positive integers. Then,

$$
\chi_{r}\left(L\left(F_{1, n}\right)\right)= \begin{cases}5, & \text { if } n=3 \leq r, \\ n, & \text { if } 1 \leq r<n, \\ n+1, & \text { if } r=n>4, \\ n+3, & \text { otherwise. }\end{cases}
$$

Proof. The case $r \neq n$ follows readily from Proposition 7. Thus, let us focus on the case $r=n$. The following three cases arise.

- Case $n=3$.

Let $c$ be a 3 -dynamic proper $\chi_{3}\left(L\left(F_{1,3}\right)\right)$-coloring. Since $d\left(u_{0} v_{0}\right)=$ $d\left(u_{0} v_{2}\right)=3$, Condition (1) implies that the set $\left\{c\left(u_{0} v_{0}\right), c\left(u_{0} v_{1}\right), c\left(u_{0} v_{2}\right)\right.$, $\left.c\left(v_{0} v_{1}\right)\right\}$ is formed by four distinct colors. In addition, since $d\left(v_{0} v_{1}\right)=3$, Condition (1), together with the fact that the map $c$ is a proper coloring, implies that $c\left(v_{1} v_{2}\right) \notin\left\{c\left(u_{0} v_{0}\right), c\left(u_{0} v_{1}\right), c\left(u_{0} v_{2}\right), c\left(v_{0} v_{1}\right)\right\}$. As a consequence, $\chi_{3}\left(L\left(F_{1,3}\right)\right)=5=\left|V\left(L\left(F_{1,3}\right)\right)\right|$.

- Case $n=4$.

Similarly to the previous case, we may ensure that

$$
c\left(v_{1} v_{2}\right) \notin\left\{c\left(u_{0} v_{0}\right), c\left(u_{0} v_{1}\right), c\left(u_{0} v_{2}\right), c\left(u_{0} v_{3}\right), c\left(v_{0} v_{1}\right), c\left(v_{2} v_{3}\right)\right\} .
$$

In addition, since $d\left(v_{1} v_{2}\right)=4$, it must be $c\left(v_{0} v_{1}\right) \neq c\left(v_{2} v_{3}\right)$. Thus, it is simply verified that $\chi_{4}\left(L\left(F_{1,4}\right)\right)=7=\left|V\left(L\left(F_{1,4}\right)\right)\right|$. 


\section{- Case $n>4$.}

From Lemma 1, we have that $n+1 \leq \chi_{n}\left(L\left(F_{1, n}\right)\right)$. In order to prove that this lower bound is reached, it is enough to define the proper $n$ coloring $c$ of the line graph $L\left(F_{1, n}\right)$ that is defined so that, for each pair of non-negative integers $j<n$ and $k<n-1$, we have that

$c\left(u_{0} v_{j}\right)=j \quad$ and $\quad c\left(v_{k} v_{k+1}\right)= \begin{cases}n, & \text { if } k \in\{0, n-2\}, \\ (k-2) \bmod n, & \text { otherwise. }\end{cases}$

Condition (1) holds and hence, $\chi_{n}\left(L\left(F_{1, n}\right)\right)=n+1$, for every positive integer $n>4$. Figure 19 illustrates the line graph $L\left(F_{1,6}\right)$.

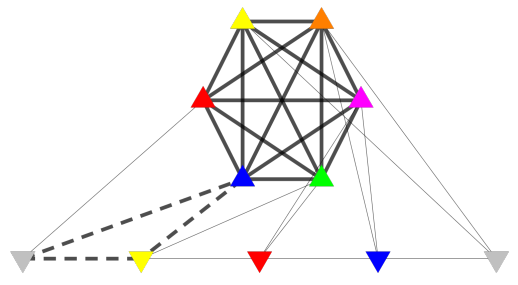

Figure 19: 6-dynamic proper 7-coloring of the line graph $L\left(F_{1,6}\right)$.

\section{Conclusion and further work}

This paper has delved into the dynamic graph coloring problem by focusing on the study of the fan graph $F_{m, n}$ and four related families of graphs: The middle graph $M\left(F_{m, n}\right)$, the total graph $T\left(F_{m, n}\right)$, the central graph $C\left(F_{m, n}\right)$ and the line graph $L\left(F_{m, n}\right)$. Particularly, we have established the exact value $\chi_{r}\left(F_{m, n}\right)$ (see Proposition 1) and sharp bounds for each one of the four mentioned families (see Propositions 2, 4, 5, 6 and 7).

Moreover, we have determined the exact $r$-dynamic chromatic number for each one of the previous four families in case of dealing with the case $m=1$ (see Theorems 1, 2, 3 and 4). In this regard, our results generalize the partial ones described in Lemma 3, which were obtained by Mohanapriya et al. in [23]. The study of exact values for the case $m>1$ is established as further work. 
Let us also remark that the fan graph $F_{m, n}$ constitutes the join product of a path of order $n$ with a set of $m$ isolated vertices. As such, this paper contributes to solve the dynamic graph coloring problem of graph products, which is receiving particular attention in the recent literature $[6,8,15,17]$. The study of the $r$-dynamic chromatic number of the join product of any two graphs is established as further work. The methodology here described may be a starting point to this end. In a similar way, the study of the dynamic

coloring problem of middle, total, central and line graphs of other types of graphs has also particular interest for the continuation of this paper.

\section{Acknowledgments}

The authors want to express their gratitude to the anonymous referees for the comprehensive reading of the paper and their pertinent comments and suggestions, which helped improve the manuscript. Further, Falcón's work is partially supported by the research project FQM-016 from Junta de Andalucía.

\section{References}

[1] Ahadi A, Akbari S, Dehghana A, Ghanbari M. On the difference between chromatic number and dynamic chromatic number of graphs. Discrete Mathematics 2012; 312 (17): 2579-2583.

[2] Akbari S, Ghanbari M, Jahanbakam S. On the dynamic chromatic number of graphs. In: Combinatorics and graphs. Contemporary Mathematics 2010; 531: 11-18.

[3] Alishahi M. On the dynamic coloring of graphs. Discrete Applied Mathematics 2011; 159 (2-3): 152-156.

[4] Alishahi M. Dynamic chromatic number of regular graphs. Discrete Applied Mathematics 2012; 160 (15): 2098-2103.

[5] Ansari N, Chandel RS, Jamal R. On b-chromatic number of prism graph families. Applications and Applied Mathematics. An International Journal (AAM) 2018; 13 (1): 286-295.

[6] Deepa T, Falcón RM, Venkatachalam M. On the $r$-dynamic coloring of the direct product of a path with either a complete graph or a wheel graph. AIMS Mathematics 2021; 6 (2): 1470-1496.

[7] Deepa T, Venkatachalam M. On $r$-dynamic coloring of the total graphs of gear graphs. Applied Mathematics E-Notes 2018; 18: 69-81. 
[8] Deepa T, Venkatachalam M, Falcón RM. On the $r$-dynamic coloring of the direct product of a path with either a path or a cycle. AIMS Mathematics 2020; 5 (6): 6496-6520.

[9] Falcón RM, Venkatachalam M, Gowri S, Nandini G, On the $r$-dynamic coloring of the direct product of a path and a $k$-subdivision of a star graph. Discrete Mathematics, Algorithms and Applications 2021; online ready: DOI: 10.1142/S1793830921501214.

[10] Furmańczyk H, Vivin JV, Mohanapriya N. r-dynamic chromatic number of some line graphs. Indian Journal of Pure and Applied Mathematics 2018; 49: 591-600.

[11] Gowri S, Venkatachalam M, Mishra VN, Mishra LN. On $r$-dynamic coloring of double star graph families. Palestine Journal of Mathematics 2021; 10: $53-62$.

[12] Hamada T, Yoshimura I. Traversability and connectivity of the middle graph of a graph. Discrete Mathematics 1976; 14 (3): 247-256.

[13] Harary F. Graph Theory. Reading, Massachusetts: Addison Wesley, 1969.

[14] Jahanbekama S, Kim J, O S, West DB. On $r$-dynamic coloring of graphs. Discrete Applied Mathematics 2016; 206: 65-72.

[15] Kaliraj K, Kumar HN, Vivin JV. On dynamic colouring of Cartesian product of complete graph with some graphs. Journal of Taibah University for Science 2020; 14 (1): 168-171.

[16] Kang R, Müller T, West DB. On $r$-dynamic coloring of grids. Discrete Applied Mathematics 2015; 186: 286-290.

[17] Kristiana AI, Utoyo MI, Alfarisi R, Dafik. $r$-dynamic coloring of the corona product of graphs. Discrete Mathematics, Algorithms and Applications 2020; 12 (2): 2050019.

[18] Lai HJ, Montgomery B, Poon H. Upper bounds of dynamic chromatic number. Ars Combinatoria 2003; 68: 193-201.

[19] Lai HJ, Montgomery B, Shui T, Fan S. Conditional colorings of graphs. Discrete Mathematics 2006; 306 (16): 1997-2004.

[20] Loeb S, Mahoney T, Reiniger B, Wise J. Dynamic coloring parameters for graphs with given genus, Discrete Applied Mathematics 2019; 235: 129-141. 
[21] Meganingtyas DEW. Analisis pewarnaan $r$-dinamis pada graf-graf khusus. PhD, Universitas Jember, UNEJ Repository, East Java, Indonesia, 2015.

[22] Mohanapriya N, Vivin JV, Venkatachalam M. $\delta$-dynamic chromatic number of helm graph families. Cogent Mathematics \& Statistics 2016; 3 (1): 1178411.

[23] Mohanapriya N, Vivin JV, Venkatachalam M. On dynamic coloring of fan graphs. International Journal of Pure and Applied Mathematics 2016; 106 (8): 169-174.

[24] Montgomery B. Dynamic coloring of graphs. PhD Thesis, West Virginia University, ProQuest LLC, Ann Arbor, MI, 2001.

[25] Nandini G, Venkatachalam M, Gowri S. On $r$-dynamic coloring of the family of bistar graphs. Communications Faculty of Sciences University of Ankara Series A1 Mathematics and Statistics 2019; 68 (1): 923-928.

[26] Nandini G, Venkatachalam M, Falcón RM. On the $r$-dynamic coloring of subdivision-edge coronas of a path. AIMS Mathematics 2020; 5 (5): 4546-4562.

[27] Praveena K, Venkatachalam M. On equitable chromatic number of tadpole graph $T_{m, n}$. Communications Faculty of Sciences University of Ankara Series A1 Mathematics and Statistics 2019; 68 (2): 1638-1646.

[28] Rajalakshmi K, Venkatachalam M. On packing coloring of helm related graphs. Journal of Discrete Mathematical Sciences and Cryptography 2019: 22 (6): 989-1005.

[29] Rohini A, Venkatachalam M. On irregular coloring of fan graph families. Journal of Physics: Conference Series 2018; 1139: 012061.

[30] Rohini A, Venkatachalam M. On irregular coloring of wheel related graphs. Communications. Faculty of Sciences. University of Ankara. Series A1. Mathematics and Statistics 2019; 68 (2): 1462-1472.

[31] Rohini A, Venkatachalam M. On irregular colorings of double wheel graph families. Communications. Faculty of Sciences. University of Ankara. Series A1. Mathematics and Statistics 2019; 68 (1): 944-949.

[32] Roy S. Packing chromatic number of certain fan and wheel related graphs. AKCE International Journal of Graphs and Combinatorics 2017; 14 (1): 63-69. 
[33] Vivin JV, Venkatachalam M. A note on $b$-coloring of fan graphs. Journal of Discrete Mathematics Sciences and Cryptography 2014; 17 (5-6): 443448.

Raúl M. FALCÓN,

Department of Applied Mathematics I,

Universidad de Sevilla,

Avenida Reina Mercedes 4 A, 41012, Sevilla, Spain.

Email: rafalgan@us.es

M. VENKATACHALAM,

Department of Mathematics,

Kongunadu Arts and Science College,

Coimbatore - 641 029, Tamil Nadu, India.

Email: venkatmaths@gmail.com

S. GOWRI

Department of Mathematics,

SNS College of Technology,

Coimbatore - 641 035, Tamil Nadu, India.

Email: gowrisathasivam@gmail.com

G. NANDINI,

Department of Mathematics,

SNS College of Technology,

Coimbatore - 641 035, Tamil Nadu, India.

Email: nandiniap2006@gmail.com 